Article

\title{
Introduction to State Estimation of High-Rate System Dynamics
}

\author{
Jonathan Hong ${ }^{1,2, *,+} \mathbb{1}$, Simon Laflamme ${ }^{2,+} \mathbb{B}^{\mathbb{D}}$, Jacob Dodson ${ }^{3,+}$ and Bryan Joyce ${ }^{4,+}$ \\ 1 Applied Research Associates, Emerald Coast Division, Niceville, FL 32578, USA \\ 2 Department of Civil, Construction, and Environmental Engineering, Iowa State University, Ames, \\ IA 50011, USA; laflamme@iastate.edu \\ 3 Air Force Research Laboratory, Munitions Directorate, Eglin Air Force Base, FL 32542, USA; \\ jacob.dodson.2@us.af.mil \\ 4 Energy Technologies and Materials, University of Dayton Research Institution, Dayton, OH 45469, USA; \\ bryan.joyce.ctr@us.af.mil \\ * Correspondence: jhong1@iastate.edu \\ + These authors contributed equally to this work.
}

Received: 8 November 2017; Accepted: 9 January 2018; Published: 13 January 2018

\begin{abstract}
Engineering systems experiencing high-rate dynamic events, including airbags, debris detection, and active blast protection systems, could benefit from real-time observability for enhanced performance. However, the task of high-rate state estimation is challenging, in particular for real-time applications where the rate of the observer's convergence needs to be in the microsecond range. This paper identifies the challenges of state estimation of high-rate systems and discusses the fundamental characteristics of high-rate systems. A survey of applications and methods for estimators that have the potential to produce accurate estimations for a complex system experiencing highly dynamic events is presented. It is argued that adaptive observers are important to this research. In particular, adaptive data-driven observers are advantageous due to their adaptability and lack of dependence on the system model.
\end{abstract}

Keywords: adaptive observers; high-rate; state estimation; dynamics; structural health monitoring

\section{Introduction}

High-rate dynamics are described as a dynamic response from a high-rate $(<100 \mathrm{~ms})$ and high-amplitude (acceleration $>100 g_{n}$ ) event such as a blast or impact. Systems which experience high-rate dynamics may undergo rapid changes that could lead to loss of economic investment or human lives [1]. There are unique challenges associated with research of structural health monitoring for systems experiencing high-rate dynamics. A system subject to high-rate dynamic environments can often experience a sudden plastic deformation, and damage can extend to the structure, electronics, and/or sensors. Rapid state estimation for these systems can be used within a feedback loop to prevent further damage and complete failure [2]. For example, rapid detection is required in the deployment of a blast mitigation system or in adapting control decisions for a hypersonic vehicle following a ballistic impact. In the problem of high-rate dynamic systems, the state estimator needs to be fast as well as robust to large uncertainties, non-stationarities, and heavy disturbances. State estimation is required when the desired states cannot be directly measured [3]. The area of research has been pioneered by Wiener [4], which led to Kalman's work [5,6]. Kalman adopted a practically structured approach, with applications found notably in the spacecrafts used in the Apollo and Polaris missions. Since then, optimal estimation research has substantially grown in popularity. In addition, advances in estimation and control theory, along with computer science, have enabled the development of observers with rapid convergence properties. Such observers have the potential to produce safer 
and smarter systems capable of responding to real-time events. In particular, the capability of an estimator to sense, analyze, and predict the heath of a system, while experiencing high-rate dynamic environments, could be invaluable for many different domains. However, this identification and adaptation task can only be conducted provided an in-time state estimation during the high-rate dynamic event, where high-rate is herein defined as the microsecond range.

The high-rate problem contains many complexities that can be summarized as having

- large uncertainties in the external loads;

- high levels of non-stationarities and heavy disturbances; and

- generated unmodeled dynamics from changes in system configuration.

A high-rate estimator must also be capable of handling these three main particularities that differentiate high-rate dynamic systems from other systems. State estimation is often required for high-rate systems because of the large levels of noise, uncertainties, and disturbances that contaminate the sensor outputs. However, the end goal of the state estimation could be different depending on the application. A popular application is damage or fault detection, in particular when a fast decision needs to be made to protect the integrity of the system, for instance a shutdown. Another application is in the adaption of physical models. For example, a different control law could be desirable for a damaged system. Whatever the application may be, state estimation is the first enabling step.

The objective of this paper is to analyze existing applications and methods of state estimation that could be useful in solving the high-rate state estimation problem for complex systems that may have nonlinear and time varying dynamics. Advantages and disadvantages of each method, with the focus on microsecond convergence rates, capable of producing accurate estimations will be surveyed. The system's observability, or how well the system can be reconstructed from measurements, is a critical concept that underlies the mathematical constructs of state estimators. It is very difficult to formulate a fully observable model of a complex physical system with high-rate system dynamics [7]. Because the focus of this paper is on the high-rate estimators' performance, we do no go into details about the observability of these complex systems. Here, attention to what aspects negatively impact convergence of estimators and the comparison between observers will give insight into how convergence rates between observers may compare. In cases when simplicity versus complexity of estimators (i.e., for computation, properties, implementation, etc.) is mentioned, the connection should be made to convergence rates in the sense that simplicity correlates to faster convergence rates. However, the performance of observers is application-specific, and the findings will be dependent on the type of scenario. The observers presented were found to be most applicable to high rate systems. The objective is to familiarize the reader with some key observer background, and gain some insight into advanced techniques to improve observer performance with respect to the high-rate state estimation problem.

The rest of the paper is organized as follows. Section 2 discusses applications where high-rate state estimation could be useful. Section 3 describes the specific challenges associated with state estimation of high-rate dynamics. Section 4 gives the background on general types of observers and their broad applications. Section 5 makes a case for adaptive observers and their application to high-rate dynamics. Section 6 concludes the paper.

\section{Applications for High-Rate State Estimation}

This section discusses engineering systems for which high-rate state estimation can be particularly useful. Examples include civil structures exposed to blast, automotive safety systems against collisions, debris strikes to space shuttles, and aerial vehicles.

\subsection{Civil Structures Exposed to Blast}

The increase in number of terrorist attacks on civilian and military structures is a growing concern. One must also consider accidental events such as gas leaks, vehicular collisions, and chemical mishaps, 
all having the potential to cause instantaneous, important changes to civil structures. These important events have the potential to jeopardize the structural integrity resulting in partial or total loss. Passive blast mitigation strategies have been developed to absorb some shock. These methods include friction elements, laminated windows, hardened concrete, etc. [8-10]. However, passive mitigation techniques are limited in performance. As an improvement, semi-active and active mitigation methods have been researched and discussed.

One example of active blast mitigation is proposed by Wadley et al. in [11]. The authors discussed using high-speed actuators to deploy pre-compressed cellular material when a blast wave is detected. Electromagnetic emission sensors are capable of detecting the blast milliseconds before the wave arrival, therefore allowing time for the actuators to react. Using this technique, the material is able to absorb the shock waves through momentum cancellation. This method offers a larger shock absorption potential over passive methods. Nevertheless, the method is not capable of adaptive actuation based on the blast dynamics. While the utilization of adaptive actuation may improve blast mitigation performance, the reliance on a control rule adds computational time, let alone the reaction time of the actuation. As an example, the time of arrival of a blast produced by a $10 \mathrm{~kg}$ Trinitrotoluene (TNT) fluctuates between 0.3 and $100 \mathrm{~ms}$ for a distance of 1 to $40 \mathrm{~m}$, requiring the sensing, estimation, and actuation process to be less than the time of arrival. In this situation, a high-rate observer would be critical in enabling the technology.

\subsection{Automotive Safety Systems against Collisions}

Fatal car accidents are on the rise [12] and improvements in our current safety measures have potential to decrease this number significantly. Current research in this field is geared toward smarter safety systems. An example is seen in [13], where the authors proposed a stereovision-based sensing method using stereo cameras and intelligent algorithms. The system classifies the occupant to determine if a child is present or if one of the passengers is in an unsafe position before deploying airbags. Although airbags play an important role in saving lives [14], this method addresses the issue that airbag systems unfortunately do sometimes cause unnecessary or even fatal injuries [15]. The child detection aspect is rather simple in that it can be done at the moment the vehicle is started. However, the unsafe position calculation is more challenging due to the fact that the calculation has to be done as an accident is occurring, in a fraction of a second [16]. The authors use a thin plate spline algorithm to boast processing times of $960 \mathrm{~ms}$ for the entire operation. It follows that smart vehicle safety systems could benefit from a high-rate observer robust to the non-stationary behaviors of the human body. It may, similar to the blast mitigation technology, enable the integration of more complex and better performing feedback systems.

\subsection{Space Shuttle and Aerial Vehicles Prone to In-Flight Anomalies}

The loss of the Columbia space shuttle in 2003 has brought light to the destructive potential of debris strikes. During the launch phase, a piece of insulating foam broke off and impacted the leading edge of the left wing while traveling at a velocity of $705 \mathrm{~m} / \mathrm{s}$ [17]. This caused a breach in the thermal protection system ultimately leading to the destruction during re-entry [18]. This catastrophe led to the research and development of the NASA Debris Radar (NDR). The NDR uses sophisticated methods to target and track debris during launch. It is also capable of automatic debris characterization assigning ballistic numbers to assess the material type, size, release location, and threat associated with the object [19]. However, the NDR is an offsite ground radar system. There are scenarios where ground intervention is not possible, for instance during communication delays or visibility limitations. Furthermore, the NDR only provides information during ascent. In the case of Columbia, the damage occurred during launch, but the loss occurred during re-entry. It follows that an onboard spacecraft structural health monitoring system could improve the in-time detectability of damage. Analogous to space shuttles, aerial vehicles often face the possibility of damage arising from impact with foreign objects including bird, hail, and lightning strikes. Any damage during flight can cause issues in 
dangerous navigational uncertainties. Because of the speed at which space shuttles and aerial vehicles travel and the rate at which damage may occur, a high-rate estimator has the potential to substantially improve safety through early detection of anomalies.

In addition, the aerospace industry is making advancements in hypersonic aerial vehicles that have the possibility of facing challenges similar to those of space shuttles. Hypersonic is defined as speeds of Mach 5 or greater [20]. Mach 5 at an altitude of $10,000 \mathrm{~m}$ and $-50{ }^{\circ} \mathrm{C}$ corresponds to $1.5 \mathrm{~km} / \mathrm{s}(0.93 \mathrm{mi} / \mathrm{s})$. In comparison, the average Boeing passenger aircraft cruises at $600 \mathrm{mph}$ or about Mach 0.8. It follows that a hypersonic vehicle travels at speeds at least six times greater than conventional passenger airplanes. At these speeds, there are possibilities for problems to quickly turn catastrophic. For instance, the distance travelled at Mach 5 is equivalent to $1.5 \mathrm{~m}(4.9 \mathrm{ft})$ over $1 \mathrm{~ms}$. A $1.5 \mathrm{MHz}$ sampling rate is necessary to obtain a $1 \mathrm{~mm}(0.04 \mathrm{in})$ travel resolution, thus requiring a high-rate observer.

\section{Challenges in State Estimation for Systems Experiencing High-Rate Dynamics}

State estimation of high-rate dynamics offers unique challenges. By definition of high-rate, estimators applicable to this research area will require rapid convergence. Take the hypothetical example of a civil structure exposed to blast equipped with an actively controlled protection system. The large uncertainties in the external loads appears in the form of lack of knowledge on the blast. The time, location, and size of the blast are all unknown. When a structure experiences a blast, it is likely to experience some damages which amount to non-stationarities and heavy disturbances. Structural damage will result in the generation of unmodeled dynamics in the form of changes in dynamic parameters, which will affect the controller performance. In this section, we describe the details of the complexities associated with high-rate systems.

\subsection{High-Rate Systems-Specific Challenges}

More precisely, high-rate systems are characterized by system complexities to include noise, uncertainty, unknown inputs, time varying parameters/states, and disturbances. Due to the high amplitude of events, noise can be introduced in many ways such as metal parts chattering at interfaces, circuit boards flexing, etc. There are many uncertainties in high-rate systems due to material response at high rates of loading being unknown [21] as well as uncertainties in boundary conditions. Unknown inputs are due to environmental influences that excite the system, and may include different dynamics, levels of chaos, and amplitudes for a single system. High-rate systems normally contain some time varying component that results from damage or changes in mechanical configuration from deformation of parts. In addition, disturbances may be present from the turbulent nature of the system which may excite resonances at the system or sensor levels. The challenges to making accurate estimations in the presence of these system complexities are briefly discussed in what follows. Conventional estimators fail at quickly estimating states when noise and uncertainties are present [22]. In practical applications, noise may arise from sensor measurements, algorithm implementation, contaminating estimation values, etc. The KF can be used to suppress noise if the noise function is appropriately built into its architecture. This noise suppression comes with added computational costs. To reduce the convergence time, the observer gain may be increased. This, however, can negatively impact the precision of the estimation as the noise can be amplified. One solution is a Uniform Robust Exact Observer (UREO) as proposed in [23]. It is tunable, and can achieve optimal convergence while maintaining precision under measurement noise.

Uncertainty is another common issue for many practical applications. For example, a major challenge for observer-driven algorithms used to estimate reaction rates in stirred tank bioreactors is in the difficulty of modeling the growth kinetics of micro-organisms [24]. Various techniques have been researched to deal with system uncertainties. For structural damage detection, methods for the formulation of objective functions using incomplete model data have been developed [25]. 
Self-tuning fusion Kalman filters based on steady-state Riccati equations are demonstrated for systems with completely unknown model parameters and noise variances [26].

There are cases for which the inputs to the system are unknown due to lack of sensors or faults in the system. For these cases, special design considerations can be made to guarantee accurate state or parameter estimations. In particular, the High Gain Observer (HGO) in [27] incorporates an auxiliary-variable to estimate unknown unsteady inputs. Reference [28] describes the usefulness of an Unknown Input Observer (UIO) for making estimations when only the output to estimate state variables is available. Originally, UIOs for a plant could be designed if and only if the plant was of minimum phase. Further development of the UIO in [28] provides alternatives to the minimum phase condition. UIO for nonlinear systems with both noise and uncertainties also require further research. One possibility is presented in [22] where a robust UIO for nonlinear systems is capable of handling both noise and uncertainty.

System models containing nonlinearities other than those from the time-varying parameters are termed parameter varying nonlinear (PVNL) systems. A sufficient condition for asymptotic convergence is developed for a two degree-of-freedoms Arcak nonlinear observer in [29]. The Arcak observer form contains two observer gain matrices of which the method is extended to optimize the second observer gain. The technique is further augmented to PVNL systems using finite dimensional relaxation method for quadratic parameter dependent Linear Matrix Inequalities (LMI).

Systems operating at high-speeds may encounter unmodeled high-order dynamics which can cause resonance, also considered as disturbances. Such resonance can lead to a decrease in estimation performance and irregular accuracy. Disturbance observers (DOB), usually used in control applications, estimate a disturbance using a low-pass filter and an inverse of the nominal model. The estimated signal is used to cancel disturbances within a system which in turn makes the system robust to uncertainties and disturbances [30]. In general, high robustness is difficult to achieve using DOB because of estimation delays attributed to pole allocation. A position-acceleration integrated disturbance observer (PAIDO) was proposed as a high-performance DOB [31]. The PAIDO structure incorporates accelerometer measurements to enlarge the frequency bandwidth of the disturbance estimation to provide better robustness [32].

Another challenge is that many of the above mentioned techniques add complexities to the estimation algorithms which slow the computational time. The decrease in computational time is another big hurdle for high-rate estimation for obvious reasons. The next section will give an example of a high-rate laboratory experiment and argue that a simple fast estimator is not sufficient for high-rate systems.

\subsection{High-Rate Dynamic Example}

An example of a high rate laboratory experiment is shown in Figure 1. Figure 1a is a picture of an MTS-66 drop tower designed to generate various impact conditions. In Figure $1 \mathrm{~b}$ is an electronics unit which consists of four circuit boards with high-g Meggitt 72 accelerometers mounted on each. These high-g accelerometers are able to accurately measure accelerations of $120,000 g_{n}$ or $120 \mathrm{~kg}_{n}$ [33], where $1 g_{n}=9.81 \mathrm{~m} / \mathrm{s}^{2}=32.2 \mathrm{ft} / \mathrm{s}^{2}$. The electronics are potted in the canister with potting material to secure all parts in place. Figure 1c shows the deceleration profile from three different tests, exhibiting varying time series behaviors for all three tests. These differences are attributed to the complex high-rate nature of the experiment.

Although the experimental setup may appear simple, this high-rate problem contains many complexities. When the drop tower is operated, the cables, which are hardwired from the accelerometers to the data acquisition system, whip violently adding noise to the measurements. Careful consideration in wires are taken to minimize the noise effect, but noise cannot be eliminated. The metal interfaces rattle upon large impacts, also adding noise. The uncertainties in this example include the unknown high-rate response of the circuit board and potting materials, and the unknown changing boundary conditions such as the bond of the potting material to the interior of the canister. 
The precise input to the system resulting from the drop tower impact is unknown. Disturbances created from sensor and/or system resonance is not obvious in these tests, however, it is not uncommon in larger impact tests.

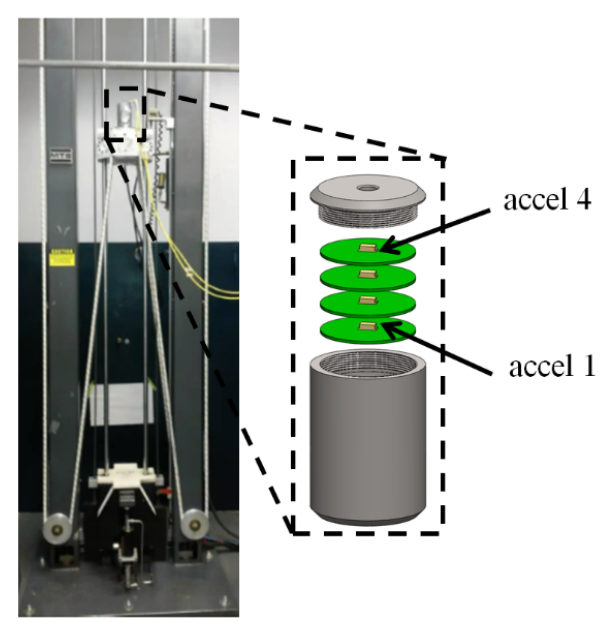

(a)

(b)

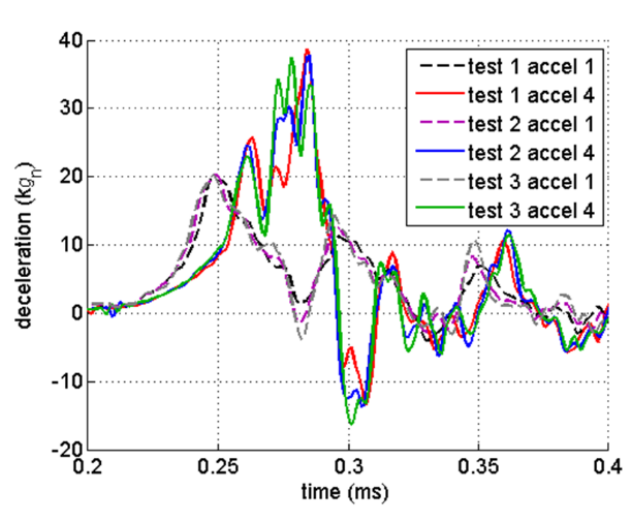

(c)

Figure 1. Experimental setup: (a) MTS-66 drop tower; (b) electronics unit; and (c) deceleration from three tests.

The deceleration event in drop tower tests typically lasts for $0.5 \mathrm{~ms}$. Due to the short duration of high-rate events, fast estimators are required. Furthermore, the estimator must be capable of handling system complexities mentioned above. In contrast, examples of fast estimators can be seen in the sensorless control of induction motors or alternating current (AC) motors, commonly used in industrial applications [34]. Different methods have been explored for estimating states of induction motors. Bodson et al. in [35] demonstrate precise motor controls in $73 \mathrm{~ms}$ using backward difference for the position measurement using low-pass filtering with a nonlinear observer constructed using a mathematical model of the system. Xu et al. in [36] show an adaptive sliding observer capable of a computation time for one update of $19 \mu \mathrm{s}$, and an Extended Kalman Filter (EKF) capable of $86 \mu$ s using a $250 \mathrm{MHz}$ processor. Zhang et al. in [37] compare the performance of a Luenberger observer (LO), sliding mode observer (SMO), and an EKF. Using a $150 \mathrm{MHz}$ processor, one update took the LO and SMO $5 \mu$ s to converge while the EKF took $100 \mu \mathrm{s}$. These observers demonstrate the capability of performance in the microsecond range appealing to the high-rate problem.

While the research efforts surveyed above represent important progress in state estimation, in most cases a particular state-estimator type addresses a specific system complexity challenge. As argued previously, the critical challenge with high-rate state estimation is associated with the presence of most of the discussed complexities in a single system. In the next section, a brief survey on observers is conducted to develop a foundation for a potential solution. The survey is intended to provide the reader with a broad background to understand the possible impact that the choice of an observer may have on systems experiencing high-rate dynamics.

\section{Background on Observers and Their General Applicability}

This section reviews typical families of observers in view of the applicability to the state-estimation problem. The most simplistic class of observers are those designed for linear and idealized systems. Such observers have, typically, fast computation time and convergence due to the triviality of computations. The Luenberger Observer (LO) [38] is a classic observer used for linear systems with well-defined numerical models. The LO has proven itself to be valuable in the areas 
of system monitoring and regulations, detecting, and identifying failures in dynamic systems [39]. However, since the LO is heavily dependent on the mathematical model of the system, disturbances, dynamic uncertainties, and nonlinearities can be particularly challenging. The KF [40] can be used in linear applications where noise is present and characterized as Gaussian [36]. The reason for the word "filter" stems from the fact that the algorithm filters through noisy data to converge to accurate estimations. While the KF typically produces estimations with higher accuracy than the LO, its implementation is more complex [37] due to possibly unmodeled nonlinearities in the system model and ill-conditioning of the covariance matrix [41]. The Sliding Mode Observer (SMO) [42] can be an alternative to provide very good robustness [43-47], but is sensitive to the choice of gain [48]. The SMO exhibits ripples in the presence of external noise [37].

Tuning of observers for linear systems can be conducted through the pole placement method. The pole placement method allows the tuner to determine observer gain matrix values based on the desired eigenvalues for system stability and convergence. The preferred location of the eigenvalues depends on the application. Generally, the further to the left in the complex plane or the more negative the real part of the pole, the faster the convergence rate. Placing the poles too far left may amplify noise [49]. The LO, KF, and SMO may exhibit high convergence rates for linear systems. However, the majority of practical problems are nonlinear and complex. For this reason, observers built for nonlinear systems are more appropriate for applications to the high-rate state estimation problem. They are discussed in what follows.

\section{Observers for Nonlinear Systems}

Observers for nonlinear systems can be classified into three main estimation methods: data-driven, statistical, and model-driven methods [50]. Popular data-driven methods include nonlinear autoregressive moving average (NARMAX) models [51], fuzzy logic [52] and neural network $(\mathrm{NN})$ estimators [53]. The performance of data-driven method is linked to the quality of data mining and interpretation algorithms, and an additional limitation can be found in the computational time required to achieve an appropriate estimate [54]. Data-driven methods were developed as tools to process information without knowledge of a system's dynamics, particularly useful for handling very complex systems. For instance, Geetha et al. [55] compared the performance of an NN and an Extended Kalman Filter (EKF) based state filter for the application to a Continuous Stirred Tank Reactor (CSTR). The performance metric indicated that the NN displayed smaller errors in the estimations over a unit sampling time than the EKF, because the CSTR had complex, nonlinear dynamics, which are difficult to characterize.

Statistical methods include Least Squares Estimator (LSE) [56], Maximum Likelihood Estimator (MLE) [57], and Particle Filters [58]. The advantage of statistical methods is the probabilistic prediction capabilities based on known parameters. These techniques can be used for nonlinear models with non-normal data. They bypass the need for linearized dynamic equations allowing global convergence of estimations. Statistical methods with simple properties such as the Least Squares method are popular in control, signal processing, and prediction theory applications. For instance, Ortega [59] discusses a method for reducing an online Least Squares parameter estimator to a set of regression vectors which guarantees a finite convergence time. The weakness of this approach is the algorithm's extreme sensitivity to noise. Franzho and Sherman [60] introduced a minimum variance spectral estimator, also known as Capon's maximum likelihood spectral estimator, that converges to a signal point spectrum even with no knowledge of the noise spectral characteristics, making it robust to contaminating noise types. Bai et al. in [61] proposed a robust statistical estimator that takes an analytic center approach for bounded error parameter estimation. The analytic center estimate minimizes the logarithmic average output error and can be implemented in a sequential form. This method comes with increased computational complexity. In [62], a Modified Recursive Least Squares estimation technique was developed for adaptive control applications were the weaker sufficiently exciting [63] condition is more likely to be satisfied than the stronger persistently exciting [64] condition. A general 
limitation of statistical methods is the reliance on available data sets for training and/or extraction of probability distribution functions.

Model-driven methods include the EKF, Unscented Kalman Filter (UKF), variations of the SMO, HGO, Nonlinear Extended State Observer (NESO), Robust State Estimators (RSE), and many more. The EKF uses a linear approximation of the nonlinear system [65] either by taking the derivative of the nonlinear function or by applying a Taylor series expansion to the desired order of the approximation. Depending on the order of the approximation, the observer is termed reduced, full, or higher order [41]. A challenge with the EKF is the linearization of the system making the corresponding propagation equations available only to the neighborhood of the estimate [66]. The EKF can be difficult to implement, difficult to tune, and highly unstable unless the system is nearly linear on the time scales of the update calculations [67]. It requires longer time for convergence compared with the LO and SMO as its computations are more complex [37]. The EKF is also difficult to apply to nonlinear systems with time varying parameters, particularly in the presence of noise [24]. The UKF uses the true nonlinear model and approximates a Gaussian distribution of the state random variable. The UKF avoids the use of complex Jacobian and Hessian matrices, making it easier to implement than the EKF with simpler computations [68]. Crassidis and Markley [69] demonstrated the superiority of the UKF with respect to the EKF in terms of accuracy, computational costs, and ease of implementation. Charles et al. [70] discussed the Utkin and Walcottzak variations of the SMO observers, both being robust estimators. However, both variations of the SMO underperform in the estimation of states when some inputs are unavailable. HGO uses a sufficiently high observer gain that will guarantee good performance of the observer in terms of accuracy and speed of convergence [71]. For most cases, the HGO is used as an LO-type estimator with large gain and the application is for estimating slowly varying states or inputs [27]. A disadvantage of the HGO is strong chattering when the gain is very large [72]. The NESO actively estimates the states, uncertainty, and unknown disturbances even when the system is unknown $[29,73]$. The RSE guarantees robustness of the designed estimator if time invariant nominal system matrices, constant filter design parameters, and stationary external inputs conditions are met. The computational complexity is comparable with that of the KFs [74].

There are other model-driven observers used to estimate nonlinear systems. To name a few, in [75], an SMO was implemented on a system that was linearized using global linearization. Ticlea and Besancon [76] discussed an immersion-based observer design where the dimension of the state is increased beyond what is done in the ESO, in order to obtain a new representation better suited for observer design. The Fokker-Planck Equations (FPE) are used to transform space and time fractional partial differential equations to a system of ordinary differential equations that are more easily solved [77], which could reduce computational time. Daum in [78] designed an exact nonlinear recursive filter where instead of linearizing nonlinear equations as conducted with the EKF, the filter solves a special class of nonlinear problems exactly with comparable computation complexity to the EKF. The coordinate transformations with output injections method is studied in [79-82]. The authors in [83] propose a dynamic observer using the Moore-Penrose generalized matrix inverse of the state matrix. The model-driven estimation method has attracted much attention because it can produce accurate state estimations when it is not possible or practical to have sensors to characterize every state [84]. Furthermore, the mathematical models required for control purposes are readily available [85]. Table 1 summarizes the observers discussed in this section in terms of general applicability to the problem of high-rate state estimation. 
Table 1. Summary of observers in terms of general applicability to the problem of high-rate state estimation.

\begin{tabular}{|c|c|c|}
\hline Observer Type & Application to High-Rate State Estimation & Reference \\
\hline Luenberger Observer (LO) & $\begin{array}{l}\text { Very fast convergence rates, but generally applies } \\
\text { to linear systems with precise nominal models, thus } \\
\text { inadequate for high-rate problem. }\end{array}$ & [39] \\
\hline Sliding Mode Observer (SMO) & $\begin{array}{l}\text { High robustness and improved results for } \\
\text { inaccurate models, but sensitive to choice of gain } \\
\text { limiting the convergence rate. }\end{array}$ & [43-48] \\
\hline Extended Kalman Filter (EKF) & $\begin{array}{l}\text { High accuracy for nonlinear systems with added } \\
\text { noise, but complex implementation leading to poor } \\
\text { convergence rates. }\end{array}$ & {$[37,41]$} \\
\hline Unscented Kalman Filter (UKF) & $\begin{array}{l}\text { Better convergence rates and higher accuracy than } \\
\text { the EKF for it uses true nonlinear model, avoids } \\
\text { complex Jacobian and Hessian matrices, and is } \\
\text { easier to implement. }\end{array}$ & [68] \\
\hline High-Gain Observer (HGO) & $\begin{array}{l}\text { Accurate and fast convergence rates for estimating } \\
\text { slowly varying states or inputs making it } \\
\text { inadequate for high-rate problems. }\end{array}$ & {$[27,71]$} \\
\hline Nonlinear Extended State Observer (NESO) & $\begin{array}{l}\text { Offers robustness to system uncertainty and } \\
\text { external disturbances. Outperformed both HGO } \\
\text { and } \mathrm{SMO} \text { in a comparative study. }\end{array}$ & {$[39,73]$} \\
\hline Robust State Estimator (RSE) & $\begin{array}{l}\text { Guarantees robustness for time invariant systems, } \\
\text { constant filter design parameters, and stationary } \\
\text { external inputs, however the convergence rate is } \\
\text { similar to Kalman Filters. }\end{array}$ & [74] \\
\hline
\end{tabular}

\section{Adaptive Observers}

A useful tool to cope with the problem of system complexities are adaptive observers (AOs). AOs can be used to estimate states and parameters using input-output measurements, ideal for handling uncertainty in state estimation [86]. They are typically characterized by asymptotic stability but slow convergence rates [87]. These observers are often modifications of the observers discussed in Section 4 that use linear transformations, such as the LO, SMO, and versions of the KF. In particular, adaptive observers have been proposed to estimate the unmeasurable states for different classes of nonlinear systems [88].

Adaptive versions of the HGO and the EKF have been studied in [89]. The high gain aspect of the HGO serves to eliminate the nonlinear part of the system, while allowing rapid convergence, thus making it a good candidate for nonlinear systems. On the other hand, the EKF filters out system or process noise, therefore advantageous for field applications. The two were combined to create a high-gain extended Kalman filter (HG-EKF) [89], which possesses the advantages of both estimators. The authors showed that the resulting HG-EKF could converge at a desired speed with the tuning of only one parameter while also being capable of noise rejection. Although promising, a key issue with the HG-EKF is with the exponential convergence occurring only at the beginning of the estimation process from the initial condition. Large perturbations in the system are difficult to estimate if they are not already modeled within the system. This issue was overcome with the development of the adaptive gain extended Kalman filter (AG-EKF) [89]. The main advantages of this observer are the noise rejection and the ability to estimate perturbations. The trade-off is that the convergence rate of the AG-EKF is slower than the HGO or HG-EKF due to the algorithm's complexity.

While showing great promise, AOs are known to have slower convergence rate. In related studies, Shahrokhi and Morari [87] attributed this problem to the utilization of single observation errors. An 
arbitrarily fast convergence rate is achieved with the use of a discrete identifier which uses multiple output errors. Global asymptotic stability is assured for sufficiently rich inputs. This method is shown to be robust and insensitive to input types and initial conditions. Khaytati and Zhu [88] identifies that complicated adaptation laws are the cause of slow convergence. As a solution, an adaptive observer with exponential parameter estimation dynamics for nonlinear systems with unmeasured regression terms is introduced, requiring Lipschitz and bounded nonlinearity constraints. In this AO configuration, a parameter can be selected as large enough to increase the rate of convergence. However, if the value is too large, it will attenuate the gain matrix. Rajamani [90] shows that the stability of the system is not based on eigenvalues alone. The eigenvalues have to be located sufficiently far left in the complex plane and the eigenvectors need to be sufficiently well-conditioned. This condition is limited in the sense that the Lipschitz constant of the nonlinear part has to be small. To obtain less conservative results, a generalized Lipschitz condition is used in conjunction with an adaptive observer for a wider class of nonlinear systems [3]. Byrski and Byrski [91] propose a modification of a modulating functions method (MFM) which enables rapid identification of step changes to parameters with minimal time delays in the estimations. There are several different solutions to increasing the rate of convergence for adaptive observers as potential candidates to high-rate estimation problems as previously discussed and summarized in Table 2.

Table 2. Adaptive observer addressed challenges and solutions to increase convergence rates.

\begin{tabular}{lll}
\hline Addressed Challenge & Solution & Reference \\
\hline Sensitivity to noise & HG-EKF & {$[89]$} \\
Sensitivity to large perturbations & AG-EKF & {$[87]$} \\
Arbitrary fast convergence & Using multiple output errors & {$[88]$} \\
Adaptation laws & Exponential parameter estimation & {$[3]$} \\
Broad applicability & Generalized Lipschitz condition & {$[91]$} \\
Fast identification of step changes & MFM & {$[$. } \\
\hline
\end{tabular}

Model-driven methods have the advantage of providing precise measures of damage due to the availability of models, therefore enabling condition assessment and system prognosis. However, they require knowledge of the physical model, which is a difficult task for real-world systems. Additionally, high-rate systems may experience changes in the structure requiring different model parameters than initially specified. Statistical methods can identify faults through a probabilistic measure, and may be used to conduct prognosis by evaluating the probability of faults, but require knowledge of probability distribution functions. The statistical properties are usually calculated from numerous tests, which is difficult to achieve for high-rate systems. Data-driven methods, in general, can provide accurate estimations based on pattern recognition and classification. Alternatively, they require precise examples and extensive training over available data set. Due to the spontaneous occurrence of high-rate events, little insight is provided in the external loads and system changes. Model- and statistics-driven methods will require significant developments to be applicable to high-rate systems.

Adaptive data-driven methods, on the other hand, can be seen as black-box systems capable of handling uncertainties found in state estimation [86]. These methods were developed as tools to process information without any knowledge of a system's dynamics. This makes data-driven methods particularly useful for dealing with very complex systems. Geetha et al. in [55] compared an NN estimator against an EKF-based state filter for continuous stirred tank reactors. The results of the study indicated smaller errors in the estimations over a unit sampling time with the use of $\mathrm{NN}$ estimators over the EKF due to the complex nonlinear dynamics difficult to characterize. DeCruyenaere et al. in [92] showed that data-driven neural networks were generally capable of outperforming KFs particularly when the system includes nonlinearities or non-Gaussian process noise. Mosavi in [93] showed that, 
while Kalman filters had higher accuracy in global positioning systems, recursive neural networks offered overall better performance due to their faster computations times.

Hybrid approaches also count advantages by combining strengths in model- and data-driven techniques. A model-driven approach can supplement data-driven methods by accounting for already understood dynamics. Conversely, data-driven methods can supplement a model-driven observer by approximating complex or difficult-to-model processes. For example, Psichogios et al. in [94] used a neural network to capture the complex, nonlinear growth rate of bacteria in a bioreactor that could then be used in a first-principles system model to find the overall biomass concentration. $\mathrm{Hu}$ et al. in [95] used a multiscale framework approach with EKF to make state-of-charge and capacity estimation. These examples demonstrate the needs for data-driven methods for complex systems. This characteristic of the adaptive data-driven method may be particularly useful for high-rate systems, which face similar challenges.

\section{Conclusions}

High-rate state estimation is a challenging task especially for complex engineering systems requiring real-time observability to ensure adequate performance. Example applications discussed civil structures exposed to blast, automotive safety systems, and space shuttle and aerial vehicles prone to in-flight anomalies. These examples highlighted the high potential of high-rate state estimators to improve the resiliency of high-rate engineering systems and save lives. The paper presented a survey of existing applications and methods for state estimation that could be useful in solving the high-rate state estimation problem.

The specific challenges associated with the high-rate problem were presented. They include large uncertainties on external loads, high levels of non-stationarities and heavy disturbances, and generations of unmodeled dynamics from mechanical changes. An induction motor study was presented to make a distinction between high-rate systems and fast systems. Additionally, a variety of observers developed to compensate for system complexities of noise, uncertainty, unknown inputs, time varying parameters/states, and disturbances to improve on performance were discussed. However, these observers do not address the combination of these challenges which forms the high-rate problem.

In providing a discussion on the suitability of various observers, the strengths and weaknesses of various methods were introduced at a broad level. Three main categories of observers were reviewed: data-driven, statistical-driven, and model-driven observers. Generally, data-driven observers are advantageous when the complexity of a system does not allow for an accurate physical representation. Statistical methods perform well when prior data is available to produce a good understanding of the statistical properties of the system's behavior. Model-driven observers produce fast and accurate estimations for systems with well defined models. The discussion extended to adaptive versions of these observers, termed adaptive observers (AOs). It was argued that given the complex nature of high-rate systems, data-driven observers have a particular promise because of the difficulty with creating a representation, and that specifically their adaptive form can be leveraged to adapt to large levels of uncertainties and complexities. However, AOs are characterized by slow convergence. Work addressing AOs' convergence has been reviewed, and it was concluded that work remains to be done in producing fast AOs.

Of particular interest is the utilization of AOs in hybrid configurations, which may take advantage of prior knowledge on a system for improved performance. They can be leveraged to replace dynamics too complex to model with a data-driven approach, possibly leading to significantly improved computational time. It follows from the discussion presented in this paper that adaptive observers and hybrid observers offer a path to developing high-rate observers capable of microsecond estimation.

Acknowledgments: The authors would like acknowledge the partial financial support from the Air Force Research Laboratory (AFRL) under the guidance of Dr. Jason Foley. Additionally, this material is based upon work partially supported by the National Science Foundation under Grant No. 1300960, the Air Force Office of Scientific Research 
(AFOSR) award number FA9550-17-1-0131, and AFRL/RWK contract number FA8651-17-D-0002. Their support is gratefully acknowledged. Any opinions, findings, and conclusions or recommendations expressed in this material are those of the authors and do not necessarily reflect the views of the United States Air Force or the National Science Foundation.

Author Contributions: The survey of observers and the initial draft of the paper was done by Jonathan Hong. Simon Laflamme provided the vision for this paper and managed the research. Jacob Dodson identified and initiated the high-rate research and provided initial funding. Laflamme, Dodson, and Joyce all put considerable work into edits and re-organization of this paper.

Conflicts of Interest: The authors declare no conflict of interest.

\section{References}

1. Lowe, R.; Dodson, J.; Foley, J. Microsecond prognostics and health management. IEEE Reliab. Soc. Newsl. 2014, 60, 1-5.

2. Connor, J.; Laflamme, S. Structural Motion Engineering; Springer: Berlin, Germany, 2014.

3. Ekramian, M.; Sheikholeslam, F.; Hosseinnia, S.; Yazdanpanah, M. Adaptive state observer for lipschitz nonlinear systems. Syst. Control Lett. 2013, 62, 319-323.

4. Gelb, A. Dual contributions of optimal estimation theory in aerospace Applications. IEEE Control Syst. Mag. 1986, 6, 3-13.

5. Kalman, R. A new approach to linear filtering and prediction results. J. Basic Eng. 1960, 82, $35-45$.

6. Grewal, M.; Andrews, A. Applications of kalman filtering in aerospace 1960 to the present. J. Basic Eng. 2010, 3, 69-78.

7. Guo, L.; Guo, Y.; Billings, S.; Coca, D. Approximate observability of infinite dimensional bilinear systems using a volterra series expansion. Syst. Control Lett. 2015, 75, 20-26.

8. Lin, L.H.; Hinman, E.; Stone, H.F.; Roberts, A.M. Survey of window retrofit solutions for blast mitigation. J. Perform. Constr. Facil. 2004, 18, 86-94.

9. Zhengqiang, Y.; Aiqun, L.; Youlin, X. Fluid viscous damper technology and its engineering application for structural vibration energy dissipation. J. Southeast Univ. (Nat. Sci. Ed.) 2002, 32, 466-473.

10. Hinman, E. Blast Safety of the Building Envelope. Whole Building Design Guide, National Institute of Building Sciences. Available online: https://www.wbdg.org/resources/blast-safety-building-envelope (accessed on 12 January 2018).

11. Wadley, H.N.; Dharmasena, K.P.; He, M.; McMeeking, R.M.; Evans, A.G.; Bui-Thanh, T.; Radovitzky, R. An active concept for limiting injuries caused by air blasts. Int. J. Impact Eng. 2010, 37, 317-323.

12. Fatal Motor Vehicle Accidents-National Summary. Transportation, 2012. Available online: http:// www2.census.gov/library/publications /2011/compendia/statab/131ed/tables/12s1105.xls (accessed on 10 January 2018).

13. Lee, S.J.; Jang, M.S.; Kim, Y.G.; Park, G.T. Stereovision-based real-time occupant classification system for advanced airbag systems. Int. J. Automot. Technol. 2011, 12, 425-432.

14. Meier, B. Study Shows Air Bags Save Lives, But Says Seat Belts Are Needed, Too. The New York Times, 26 June 1992. Available online: http:/ / www.nytimes.com/1992/06/26/us/study-shows-air-bags-save-lives-butsays-seat-belts-are-needed-too.html?pagewanted=all (accessed on 12 January 2018).

15. Cunningham, K.; Brown, T.; Gradwell, E.; Nee, P. Airbag associated fatal head injury: Case report and review of the literature on airbag injuries. J. Accid. Emerg. Med. 2000, 17, 139-142.

16. Chiru, M. Injuries of the foot and ankle joint and their mechanisms. In Proceedings of the Fisita World Automotive Congress, Barcelona, Spain, 23-27 May 2004; pp. 1-13.

17. Walker, J.D. From columbia to discovery: Understanding the impact threat to the space shuttle. Int. J. Impact Eng. 2009, 36, 303-317.

18. Smith, D. Megalightning and The Demise of STS-107 Space Shuttle Columbia: A Fresh Look at the Available Evidence. 2009. Available online: http:/ / www.columbiadisaster.info (accessed on 10 January 2018).

19. Kent, B.M. Return-to-Flight Electromagnetic Measurements-The NASA shuttle ascent debris radar system. In Proceedings of the IEEE 8th European Conference on Antennas and Propagation, The Hague, The Netherlands, 6-11 April 2014; pp. 1-3.

20. Hyersonic. Available online: http://www.merriam-webster.com (accessed on 10 January 2018). 
21. Kolsky, H. An investigation of the mechanical properties of materials at very high rates of loading. Proce. Phys. Soc. Sect. B 1949, 62, 676.

22. Mondal, S.; Chakraborty, G.; Bhattacharyya, K. Robust unknown input observer for nonlinear systems and its application to fault detection and isolation. J. Dyn. Syst. Meas. Control 2008, 130, 1-5.

23. Fraguela, L.; Angulo, M.T.; Moreno, J.; Fridman, L. Design of a Prescribed Convergence Time Uniform Robust Exact Observer in the Presence of Measurement Noise. In Proceedings of the IEEE 51st Annual Conference on Decision and Control, Maui, HI, USA, 10-13 December 2012; pp. 6615-6620.

24. Oliveira, R.M.F.; Ferreira, E.; Oliveira, F.; Azevedo, S. A Study on the convergence of observer-based kinetics estimators in stirred tank bioreactors. Korean Inst. Chem. Eng. 1994, 6, 367-371.

25. Kourehli, S.S.; Bagheri, A.; Amiri, G.G.; Ghafory-Ashtiany, M. Structural damage detection using incomplete modal data and incomplete static response. KSCE J. Civ. Eng. 2013, 17, 216-223.

26. Tao, G.L.; Deng, Z.l. Convergence of Self-Tuning Riccati Equation for Systems with Unknown Parameters and Noise Variances. In Proceedings of the IEEE 8th World Congress on Intelligent Control and Automation (WCICA), Jinan, China, 7-9 July 2010; pp. 5732-5736.

27. Stricker, K.; Kocher, L.; Van Alstine, D.; Shaver, G.M. Input observer convergence and robustness: Application to compression ratio estimation. Control Eng. Pract. 2013, 21, 565-582.

28. Yamada, K.; Kobayashi, M. A Design Method for Unknown Input Observer for Non-Minimum Phase Systems. In Proceedings of the International Workshop and Conference on Photonics and Nanotechnology, International Society for Optics and Photonics, Gifu, Japan, 7 January 2007; pp. 1-6.

29. Wang, Y.; Rajamani, R.; Bevly, D.M. Observer Design for Differentiable Lipschitz Nonlinear Systems with Time-Varying Parameters. In Proceedings of the IEEE 53rd Annual Conference on Decision and Control (CDC), Los Angeles, CA, USA, 15-17 December 2014; pp. 145-152.

30. Kim, B.K.; Chung, W.K.; Ohba, K. Design and performance tuning of sliding-mode controller for high-speed and high-accuracy positioning systems in disturbance observer framework. IEEE Trans. Ind. Electron. 2009, 56, 3798-3809.

31. Yoshioka, T.; Phuong, T.T.; Yabuki, A.; Ohishi, K.; Miyazaki, T.; Yokokura, Y. High-performance load torque compensation of industrial robot using kalman-filter-based instantaneous state observer. IEEE J. Ind. Appl. 2015, 4, 589-590.

32. Katsura, S.; Irie, K.; Ohishi, K. Wideband force control by position-acceleration integrated disturbance observer. IEEE Trans. on Ind. Electron. 2008, 55, 1699-1706.

33. Beliveau, A.; Hong, J.; Coker, J.; Glikin, N. COTS piezoresistive shock accelerometers performance evaluation. Shock Vib. Exch. 2012, 83.

34. Shahnazi, R.; Zhao, Q.; Sari, A.H.A.; Jeinsch, T. Dynamic Nonlinear Unknown Input Observer for Fault Detection of Induction Motors. In Proceedings of the 23rd Iranian Conference on Electrical Engineering (ICEE), Tehran, Iran, 10-14 May 2015; pp. 823-828.

35. Bodson, M.; Chiasson, J.; Novotnak, R.T. Nonlinear speed observer for high-Performance induction motor control. IEEE Trans. on Ind. Electron. 1995, 42, 337-343.

36. Xu, Z.; Rahman, F.; Xu, D. Comparative Study of an Adaptive Sliding Observer and an EKF for Speed Sensor-Less DTC IPM Synchronous Motor Drives. In Proceedings of the IEEE Power Electronics Specialists Conference, Orlando, FL, USA, 17-21 June 2007; pp. 2586-2592.

37. Zhang, Y.; Zhao, Z.; Lu, T.; Yuan, L.; Xu, W.; Zhu, J. A Comparative Study of Luenberger Observer, Sliding Mode Observer and Extended Kalman Filter for Sensorless Vector Control of Induction Motor Drives. In Proceedings of the IEEE Energy Conversion Congress and Exposition, San Jose, CA, USA, 20-24 September 2009; pp.2466-2473.

38. Luenberger, D. An introduction to observers. IEEE Trans. Autom. Control 1971, 16, 596-602.

39. Wang, W.; Gao, Z. A Comparison Study of Advanced State Observer Design Techniques. In Proceedings of the IEEE American Control Conference, Denver, CO, USA, USA, 4-6 June 2003; Volume 6, pp. 4754-4759.

40. Kalman, R.E.; Bucy, R.S. New results in linear filtering and prediction theory. J. Fluids Eng. 1961, 83, 95-108.

41. Daum, F.E. Nonlinear filters: Beyond the kalman filter. IEEE Aerosp. Electron. Syst. Mag. 2005, 20, 57-69.

42. Drakunov, S.; Utkin, V. Sliding Mode Observers. Tutorial. In Proceedings of the 34th IEEE Conference on Decision and Control, New Orleans, LA, USA, 13-15 December 1995; Volume 4, pp. 3376-3378.

43. Li, J.; Xu, L.; Zhang, Z. An adaptive sliding-mode observer for induction motor sensorless speed control. IEEE Trans.Ind. Appl. 2005, 41, 1039-1046. 
44. Lascu, C.; Andreescu, G.D. Sliding-mode observer and improved integrator with DC-Offset compensation for flux estimation in sensorless-controlled induction motors. IEEE Trans. Ind. Electron. 2006, 53, 785-794.

45. Tursini, M.; Petrella, R.; Parasiliti, F. Adaptive sliding-mode observer for speed-sensorless control of induction motors. IEEE Trans. Ind. Appl. 2000, 36, 1380-1387.

46. Utkin, V.; Guldner, J.; Shi, J. Sliding Mode Control in Electro-Mechanical Systems; CRC press: Boca Raton, FL, USA, 2009.

47. Yongchang, Z.; Zhengming, Z.; Yingchao, Z.; Gaosheng, S. A Full Sliding Mode Sensorless Control of Three-Level Inverter-Fed Induction Motors. In Proceedings of the IEEE Power Electronics Specialists Conference, Rhodes, Greece, 15-19 June 2008; pp. 2825-2831.

48. Chen, X.; Shen, W.; Cao, Z.; Kapoor, A. A Comparative Study of Observer Design Techniques for State of Charge Estimation in Electric Vehicles. In Proceedings of the 7th IEEE Conference on Industrial Electronics and Applications (ICIEA), Singapore, 18-20 July 2012; pp. 102-107.

49. Li, P. State Feedback; University of Minnesota: Minneapolis, MN, USA, 2008, pp. 98-136.

50. Xu, J.; Lum, K.Y.; Xie, L.; Loh, A.P. Fault detection and isolation of nonlinear systems: An unknown input observer approach with sum-of-squares techniques. J. Dyn. Syst. Meas. Control 2012, 134, 1-7.

51. Billings, S. Nonlinear System Identification: NARMAX Methods in the Time, Frequency, and Spatio-Temporal Domains; John Wiley and Sons: Hoboken, NJ, USA, 2013.

52. Zadeh, L.A. A Summary and Update of "Fuzzy Logic". In Proceedings of the IEEE International Conference on Granular Computing (GrC), San Jose, CA, USA, 14-16 August 2010, pp. 42-44.

53. Derrida, B. Introduction to neural network models. Nucl. Phys. B 1988, 4, 673-677.

54. Guyon, I. Neural networks and applications tutorial. Phys. Rep. 1991, 207, 215-259.

55. Geetha, M.; Jerome, J.; Kumar, P.A.; Anadhan, K. Comparative Performance Analysis of Extended Kalman Filter and Neural Observer for State Estimation of Continuous Stirred Tank Reactor. In Proceedings of the IEEE Fourth International Conference on Computing, Communications and Networking Technologies, Tiruchengode, India, 4-6 July 2013; pp. 1-7.

56. Swerling, P. Modern state estimation methods from the viewpoint of the method of least squares. IEEE Trans. Autom. Control 1971, 16, 707-719.

57. Myung, I.J. Tutorial on maximum likelihood estimation. J. Mathe. Psychol. 2003, 47, 90-100.

58. Gordon, N.J.; Salmond, D.J.; Smith, A.F. Novel approach to nonlinear/non-gaussian bayesian state estimation. IEEE Proce. Radar Signal Process. 1993, 140, 107-113.

59. Ortega, R. An on-line least-squares parameter estimator with finite convergence time. Proc. IEEE 1988, 76, 847-848.

60. Frazho, A.E.; Sherman, P.J. On the Convergence of the Minimum Variance Spectral Estimator in Nonstationary Noise. In Proceedings of the IEEE International Conference on Acoustics, Speech, and Signal Processing, Toronto, ON, Canada, 14-17 April 1991, pp. 3141-3143.

61. Bai, E.W.; Fu, M.; Tempo, R.; Ye, Y. Convergence results of the analytic center estimator. IEEE Trans. Autom. Control 2000, 45, 569-572.

62. Kamen, E. A recursive parameter estimator yielding exponential convergence under sufficient excitation. Syst. Signal Process. Circuits 1989, 8, 207-228.

63. Johnson, C.R. Lectures on Adaptive Parameter Estimation; Prentice-Hall, Inc.: Upper Saddle River, NJ, USA, 1988.

64. Bitmead, R.R.; Anderson, B. Lyapunov techniques for the exponential stability of linear difference equations with random coefficients. IEEE Trans. Autom. Control 1980, 25, 782-787.

65. Song, Y.; Grizzle, J.W. The extended Kalman filter as a local asymptotic observer for nonlinear discrete-time systems. In Proceedings of the IEEE American Control Conference, Chicago, IL, USA, 24-26 June 1992; pp. 3365-3369.

66. Boutayeb, M.; Rafaralahy, H.; Darouach, M. Convergence analysis of the extended kalman filter used as an observer for nonlinear deterministic discrete-time systems. IEEE Trans. Autom. Control 1997, 42, 581-586.

67. Julier, S.; Uhlmann, J. New extension of the Kalman filter to nonlinear systems. Signal processing, sensor fusion, and target recognition VI. AeroSense'97 1997, 3068, 182-193.

68. Zhang, H.; Dai, G.; Sun, J.; Zhao, Y. Unscented kalman filter and its nonlinear application for tracking a moving target. Opt.-Int. J. Light Electron Opt. 2013, 124, 4468-4471. 
69. Crassidis, J.L.; Markley, F.L. Unscented filtering for spacecraft attitude estimation. J. Guid. Control Dyn. 2003, $26,536-542$.

70. Charles, A.; Nandhini, M.; Sakthivel, A. A comparative study of sliding mode observers. Int. J. Recent Innov. Trends Comput. Commun. 2015, 3, 2923-2929.

71. Khalil, H.K.; Praly, L. High-gain observers in nonlinear feedback control. Int. J. Robust Nonlinear Control 2014, 24, 993-1015.

72. Braun, T.; Strausberger, F.; Reuter, J. State Estimation for Fast-Switching Solenoid Valves: A Study On Practical Nonlinear Observers and New Experimental Results. In Proceedings of the IEEE 20th International Conference on Methods and Models in Automation and Robotics (MMAR), Miedzyzdroje, Poland, 24-27 August 2015; pp. 862-867.

73. Mahapatro, K.A.; Chavan, A.D.; Suryawanshi, P.V.; Rane, M.E. Comparative Analysis of Linear and Non-linear Extended State Observer with Application to Motion Control. In Proceedings of the International Conference on Convergence of Technology, Pune, India, 6-8 April 2014; pp. 1-7.

74. Zhou, T. On the convergence and stability of a robust state estimator. IEEE Trans. Autom. Control 2010, 55, 708-714.

75. Walcott, B.; Corless, M.; Żak, S. Comparative study of non-linear state-observation techniques. Int. J. Control 1987, 45, 2109-2132.

76. Țiclea, A.; Besançon, G. Immersion-Based Observer Design. In Nonlinear Observers and Applications; Springer: Berlin, Germany, 2007; pp. 115-138.

77. Liu, F.; Anh, V.; Turner, I. Numerical solution of the space fractional fokker-planck equation. J. Comput. Appl. Math. 2004, 166, 209-219.

78. Daum, F.E. New Exact Nonlinear Filters: Theory and applications. In Proceedings of the SPIE's International Symposium on Optical Engineering and Photonics in Aerospace Sensing, Orlando, FL, USA, 6 July 1994; pp. 636-649.

79. Bastin, G.; Gevers, M.R. Stable adaptive observers for nonlinear time-varying systems. IEEE Trans. Autom. Control 1988, 33, 650-658.

80. Marino, R. Adaptive observers for single output nonlinear systems. IEEE Trans. Autom. Control 1990, 35, 1054-1058.

81. Marino, R.; Tomei, P. Global adaptive observers for nonlinear systems via filtered transformations. IEEE Trans. Autom. Contro 1992, 37, 1239-1245.

82. Marino, R.; Tomei, P. Nonlinear Control Design: Geometric, Adaptive and Robust; Prentice Hall International (UK) Ltd.: London, UK, 1996.

83. Samanta, R.; Das, G. A Comparative Study Between Luenberger Full Order Observer and Full Order Observer Designed by Generalized Matrix Inverse Method. In Proceedings of the IEEE First International Conference on Automation, Control, Energy and Systems (ACES), Hooghy, India, 1-2 Feburary 2014; pp. 1-5.

84. Besançon, G. An Overview on Observer Tools for Nonlinear Systems. In Nonlinear Observers and Applications; Springer: Berlin, Germany, 2007; pp. 1-33.

85. Zarei, J.; Shokri, E. Robust sensor fault detection based on nonlinear unknown input observer. Measurement 2014, 48, 355-367.

86. Yingjuan, Y.; Pengzhang, X. Design of a Nonlinear Adaptive Observer for a Class of Lipschitz Systems. In Proceedings of the IEEE 33rd Chinese Control Conference (CCC), Nanjing, China, 28-30 July 2014; pp. 2240-2243.

87. Shahrokhi, M.; Morari, M. A discrete adaptive observer and identifier with arbitrarily fast rate of convergence. IEEE Trans. Autom. Control 1982, 27, 506-509.

88. Khayati, K.; Zhu, J. Adaptive Observer for a Large Class of Nonlinear Systems with Exponential Convergence of Parameter Estimation. In Proceedings of the IEEE International Conference on Control, Decision and Information Technologies (CoDIT), Hammamet, Tunisia, 6-8 May 2013; pp. 100-105.

89. Boizot, N.; Busvelle, E. Adaptive-Gain Observers and Applications. In Nonlinear Observers and Applications; Springer: Berlin, Germany, 2007; pp. 71-114.

90. Rajamani, R. Observers for lipschitz nonlinear systems. IEEE Trans. Autom. Control 1998, 43, 397-401.

91. Byrski, W.; Byrski, J. On-Line Fast Identification Method and Exact State Observer for Adaptive Control of Continuous System. In Proceedings of the IEEE 11th World Congress on Intelligent Control and Automation (WCICA), Shenyang, China, 29 June-4 July 2014; pp. 4491-4497. 
92. DeCruyenaere, J.; Hafez, H. A Comparison between Kalman Filters and Recurrent Neural Networks. In Proceedings of the IEEE International Joint Conference on Neural Networks, Baltimore, MD, USA, 7-11 June 1992; Volume 4, pp. 247-251.

93. Mosavi, M. Comparing DGPS corrections prediction using neural network, fuzzy neural network, and kalman filter. GPS Solut. 2006, 10.

94. Psichogios, D.C.; Ungar, L.H. A hybrid neural network-first principles approach to process modeling. AIChE J. 1992, 38, 1499-1511.

95. Hu, C.; Youn, B.; Chung, J. A multiscale framework with extended kalman filter for lithium-ion battery SOC and capacity estimation. Appl. Energy 2012, 92, 694-704.

(C) 2018 by the authors. Licensee MDPI, Basel, Switzerland. This article is an open access article distributed under the terms and conditions of the Creative Commons Attribution (CC BY) license (http://creativecommons.org/licenses/by/4.0/). 